\title{
Safety and Efficacy of INCB018424, a JAK1 and JAK2 Inhibitor, in Myelofibrosis
}

\author{
Srdan Verstovsek, M.D., Ph.D., Hagop Kantarjian, M.D., Ruben A. Mesa, M.D., Animesh D. \\ Pardanani, M.B., B.S., Ph.D., Jorge Cortes-Franco, M.D., Deborah A. Thomas, M.D., Zeev \\ Estrov, M.D., Jordan S. Fridman, Ph.D., Edward C. Bradley, M.D., Susan Erickson-Viitanen, \\ Ph.D., Kris Vaddi, Ph.D., Richard Levy, M.D., and Ayalew Tefferi, M.D. \\ M.D. Anderson Cancer Center, Houston (S.V., H.K., J.C.-F., D.A.T., Z.E.); Mayo Clinic, Scottsdale, \\ AZ (R.A.M.); Mayo Clinic, Rochester, MN (A.D.P., A.T.); and Incyte, Wilmington, DE (J.S.F., \\ E.C.B., S.E.-V., K.V., R.L.)
}

\section{Abstract}

Background-Myelofibrosis is a Philadelphia chromosome-negative myeloproliferative neoplasm associated with cytopenias, splenomegaly, poor quality of life, and shortened survival. About half of patients with myelofibrosis carry a gain-of-function mutation in the Janus kinase 2 gene $(J A K 2 \mathrm{~V} 617 \mathrm{~F})$ that contributes to the pathophysiology of the disease. INCB018424 is a potent and selective Janus kinase 1 (JAK1) and JAK2 inhibitor.

Methods-We conducted a phase 1-2 trial of INCB018424 in patients with JAK2 V617Fpositive or $J A K 2 \mathrm{~V} 617 \mathrm{~F}$-negative primary myelofibrosis, post-essential thrombocythemia myelofibrosis, or post-polycythemia vera myelofibrosis.

\begin{abstract}
Results-A total of 153 patients received INCB018424 for a median duration of more than 14.7 months. The initial dose-escalation phase established $25 \mathrm{mg}$ twice daily or $100 \mathrm{mg}$ once daily as maximum tolerated doses, on the basis of reversible thrombocytopenia. A dose-dependent suppression of phosphorylated signal transducer and activator of transcription 3 (STAT3), a marker of JAK signaling, was demonstrated in patients with wild-type $J A K 2$ and in patients with the JAK2 V617F mutation. We studied additional doses and established that a $15-\mathrm{mg}$ twice-daily starting dose, followed by individualized dose titration, was the most effective and safest dosing regimen. At this dose, 17 of 33 patients (52\%) had a rapid objective response ( $250 \%$ reduction of splenomegaly) lasting for 12 months or more, and this therapy was associated with grade 3 or grade 4 adverse events (mainly myelosuppression) in less than $10 \%$ of patients. Patients with debilitating symptoms, including weight loss, fatigue, night sweats, and pruritus, had rapid improvement. Clinical benefits were associated with a marked diminution of levels of circulating inflammatory cytokines that are commonly elevated in myelofibrosis.
\end{abstract}

Conclusions-INCB018424 was associated with marked and durable clinical benefits in patients with myelofibrosis for whom no approved therapies existed.

Address reprint requests to Dr. Verstovsek at the Leukemia Department, M.D. Anderson Cancer Center, Unit 428, 1515 Holcombe Blvd., Houston, TX 77030, or at sverstov@mdanderson.org.

No other potential conflict of interest relevant to this article was reported. 
Myelofibrosis is manifested as primary myelofibrosis, post-essential thrombocythemia myelofibrosis, or post-polycythemia vera myelofibrosis and is characterized by clinical signs (e.g., progressive anemia, bone marrow fibrosis, and splenomegaly) and a constellation of debilitating symptoms (fatigue, weakness, bone pain, a hypercatabolic state, and weight loss). ${ }^{1}$ Survival in myelofibrosis is related to the number of risk factors and ranges from 2 to 4 years among patients with two or more risk factors (intermediate- 2 or high risk) to 8 to 11 years among patients with no risk factors or one risk factor (intermediate-1 or low risk) (see Table 1A in the Supplementary Appendix, available with the full text of this article at NEJM.org). ${ }^{2-4}$ Conventional drugs used to treat myelofibrosis provide only palliative benefits and are not approved for this indication. ${ }^{5}$

The discovery of $J A K 2 \mathrm{~V} 617 \mathrm{~F}$, a somatic gain-of-function mutation in the Janus kinase 2 (JAK2) in classic Philadelphia chromosome ( $\mathrm{Ph}$ )-negative myeloproliferative neoplasms, which include essential thrombocythemia, polycythemia vera, and primary myelofibrosis, generated interest in the development of JAK2-targeted therapies for these diseases. ${ }^{6,7}$ The $J A K 2 \mathrm{~V} 617 \mathrm{~F}$ mutation is present in approximately $50 \%$ of patients with myelofibrosis.

Some clinical signs of the disease, such as anemia and splenomegaly, and the risk of transformation to acute myeloid leukemia (AML) have been related to $J A K 2 \mathrm{~V} 617 \mathrm{~F}$ mutational status or the $J A K 2 \mathrm{~V} 617 \mathrm{~F}$ allele burden. ${ }^{8-10}$ Other mutations in the $J A K 2$ and $M P L$ (thrombopoietin receptor) genes that also result in an exaggerated JAK2 signaling ${ }^{11}$ collectively point to unchecked activation of JAK2 as a potential mechanistic cause of $\mathrm{Ph}$ negative myeloproliferative neoplasms. INCB018424, a potent, selective inhibitor of JAK1 and JAK2, has been shown to be effective in preclinical studies in models of myeloproliferative neoplasms. ${ }^{12}$ We describe the clinical evaluation of a JAK1 and JAK2 inhibitor in myelofibrosis.

\section{Methods}

\section{Eligibility Criteria}

Patients 18 years of age or older with primary myelofibrosis, post-essential thrombocythemia myelofibrosis, or post-polycythemia vera myelofibrosis (according to World Health Organization criteria revised in 2001) ${ }^{13}$ were eligible for enrollment if they required therapy, had had a relapse, or had severe side effects from therapy or disease that was refractory to previous therapy. Patients with newly diagnosed disease were eligible if they had intermediate- or high-risk disease according to the Lille scoring system. ${ }^{14}$ (The Lille system defines risk according to the number of adverse prognostic factors [a hemoglobin level of $<10 \mathrm{~g}$ per deciliter, or a white-cell count of $<4 \times 10^{9}$ or $>30 \times 10^{9}$ per liter]: 0 [low risk], 1 [intermediate risk], and 2 [high risk].) Patients with newly diagnosed disease also were eligible if they had symptomatic splenomegaly (or hepatomegaly in patients who had undergone splenectomy) that was palpable $10 \mathrm{~cm}$ or more below the left costal margin.

Additional eligibility criteria included an Eastern Cooperative Oncology Group (ECOG) performance status of 2 or less. ${ }^{15}$ (The ECOG performance status is graded on a scale of 0 to 5 , with a higher score indicating a greater severity of illness. A score of 0 indicates that the patient is fully active and able to carry out all activities without restriction, a score of 1 
indicates that the patient is restricted in physically strenuous activity but ambulatory and able to carry out work of a light or sedentary nature [e.g., light housework or office work], and a score of 2 indicates that the patient is ambulatory and capable of all self-care but is unable to carry out any work activities and is out of bed more than $50 \%$ of waking hours.)

Other eligibility criteria included an absolute neutrophil count of more than $1.5 \times 10^{9}$ per liter, a platelet count of more than $100 \times 10^{9}$ per liter, a bilirubin level of $2.0 \mathrm{mg}$ per deciliter (34 $\mu \mathrm{mol}$ per liter) or less, an alanine aminotransferase level of 2.5 times the upper limit of the normal range or less, and a creatinine level of $2.5 \mathrm{mg}$ per deciliter ( $220 \mu \mathrm{mol}$ per liter) or less. Patients could not have received therapy for myelofibrosis for 2 weeks before enrollment, and they could not have received busulfan or pegylated interferon for 4 weeks before enrollment. A negative serum pregnancy test was required in women of child-bearing potential, and effective contraception in all men and women of child-bearing potential was mandatory. Patients were excluded if they were pregnant or nursing, if they had another malignant condition (except nonmelanoma skin cancer or cervical intraepithelial neoplasia) or known active hepatitis infection, if they were positive for the human immunodeficiency virus, or if they were receiving glucocorticoid therapy (equivalent to prednisone at a dose of $>10 \mathrm{mg}$ per day) or if therapy with cytochrome P450 (CYP3A4 and CYP1A2) inhibitors or inducers was being administered or was planned.

\section{Study Design}

This phase 1-2 study was approved by the institutional review boards of the M.D. Anderson Cancer Center and Mayo Clinic and was conducted in accordance with the principles of the Declaration of Helsinki. All patients provided written informed consent. The sponsor of the study, Incyte, supplied INCB018424 tablets that were administered orally in an outpatient setting. Both once-daily and twice-daily dose schedules were evaluated in the phase 1 part of the study with the use of a standard $3+3$ cohort design ( 3 to 6 patients per dose cohort) to determine safety and tolerability and to establish dose-limiting toxicity and the maximum tolerated dose. On the basis of an expanded cohort of 27 patients in the group of patients who received the maximum tolerated dose, it was predicted that there would be a greater than $90 \%$ chance of one or more adverse events, with a $10 \%$ underlying prevalence rate. Similarly, if the response rate was $10 \%$ or greater, the probability of one response or more would be more than $90 \%$. Several alternative twice-daily dose schedules (below the maximum tolerated dose) were studied in the phase 2 part of the study. Four weeks of daily oral therapy was considered to constitute one cycle.

Patients were followed for a median of 14.7 months. The response was evaluated with the use of the response criteria of the International Working Group for Myelofibrosis Research and Treatment (Table 1B in the Supplementary Appendix). The treatment plan, response assessment, and laboratory correlates are described in the Supplementary Appendix.

The first author and the sponsor of the study wrote the first draft of the manuscript, and all of the authors made the decision to submit the article for publication. All authors vouch for the accuracy and completeness of the reported data. The authors also certify that the study as reported conforms with the protocol (for details, see the protocol, available at NEJM.org). 


\section{Results}

\section{Patients and Dose Schedules}

A total of 153 patients with advanced primary myelofibrosis, post-essential thrombocythemia myelofibrosis, or post-polycythemia vera myelofibrosis were enrolled (Table 1, and Table 2 in the Supplementary Appendix). The starting dose of INCB018424 was $25 \mathrm{mg}$ twice daily followed by escalation to $50 \mathrm{mg}$ twice daily. Subsequently, oncedaily doses were also evaluated, from $25 \mathrm{mg}$ up to $200 \mathrm{mg}$ once daily. Thrombocytopenia was identified as the dose-limiting toxic effect, and $25 \mathrm{mg}$ twice daily and $100 \mathrm{mg}$ once daily were identified as the maximum tolerated doses. However, 2 of 6 patients who received $100 \mathrm{mg}$ once daily had clinically significant thrombocytopenia during the second month of therapy; therefore, additional patients were added to the cohorts of patients who received 25 $\mathrm{mg}$ twice daily and $50 \mathrm{mg}$ once daily.

In the phase 2 part of the study, additional dosing schedules were studied in succession, with defined enrollment targets and additional evaluations (Fig. 1 in the Supplementary Appendix) to identify an effective dosing schedule with a reduced incidence of thrombocytopenia. These schedules were as follows: $25 \mathrm{mg}$ twice daily, with a reduction to $10 \mathrm{mg}$ twice daily after two cycles ( 2 months) of therapy (induction-maintenance); $10 \mathrm{mg}$ twice daily, with allowance for dose escalation after three cycles (3 months) if there was no response and no toxicity; and $10 \mathrm{mg}$ twice daily (in patients with baseline platelet counts of $100 \times 10^{9}$ to $200 \times 10^{9}$ per liter) or $15 \mathrm{mg}$ twice daily (in patients with baseline platelet counts of $>200 \times 10^{9}$ per liter), with allowance for dose increases (monthly, 5-mg twice-daily increments) up to $25 \mathrm{mg}$ twice daily, if there was no response and no toxicity (individualized dose adjustment). The baseline characteristics of the patients in these groups were well balanced (Table 2 in the Supplementary Appendix).

\section{Response}

According to the response criteria of the International Working Group for Myelofibrosis Research and Treatment (hereafter called the International Working Group) (Table 1B in the Supplementary Appendix), ${ }^{16} 61$ of 140 patients with an enlarged spleen at study entry (44\%) had an objective response (clinical improvement), based on a reduction of $50 \%$ or more in palpable splenomegaly within the first 3 months after therapy. The response rate was highest among the 33 patients who received $15 \mathrm{mg}$ and the 39 patients who received $25 \mathrm{mg}$ twice daily ( $52 \%$ and $49 \%$, respectively), intermediate among the 22 patients who received $50 \mathrm{mg}$ once daily (41\%), and lowest among the 27 patients who received $10 \mathrm{mg}$ twice daily (30\%) (Fig. 1A). None of the patients who received $25 \mathrm{mg}$ once daily had clinical improvement. Clinical improvement was observed in 3 of 4 patients receiving $50 \mathrm{mg}$ twice daily, 3 of 6 patients receiving $100 \mathrm{mg}$ once daily, and 2 of 3 patients receiving $200 \mathrm{mg}$ once daily, but these patients required dose reductions because of thrombocytopenia. Among patients who had a response according to the International Working Group criteria, the response was maintained after 12 months of therapy, in $71 \%$ of patients who received $10 \mathrm{mg}$ twice daily, $73 \%$ of patients who received $15 \mathrm{mg}$ twice daily, $78 \%$ of patients who received $25 \mathrm{mg}$ twice daily, and $75 \%$ of patients who received $50 \mathrm{mg}$ once daily. 
The majority of patients ( $70 \%$ of patients who received $10 \mathrm{mg}$ twice daily, $82 \%$ of patients who received $15 \mathrm{mg}$ twice daily, and $77 \%$ of patients who received $25 \mathrm{mg}$ twice daily) had a $25 \%$ or greater reduction in spleen size while receiving therapy (Fig. 1B). This reduction occurred within the first 1 to 2 months of therapy, was durable beyond 1 year of therapy in all 16 patients for whom data were available at 1.5 years and all 8 patients for whom data were available at 2 years (Fig. 1C), and was similar among patients who received $15 \mathrm{mg}$ twice daily (with individualized dose adjustment for an inadequate response and no toxicity) and those who received $25 \mathrm{mg}$ twice daily (based on the intention-to-treat analysis) (Fig. 1D). Although the best response was noted in the subgroup of patients who started with and continued to receive $25 \mathrm{mg}$ twice daily, $60 \%$ of the patients who started with $25 \mathrm{mg}$ twice daily required dose reductions because of thrombocytopenia; this suggests an advantage of initiating therapy at a dose of $15 \mathrm{mg}$ twice daily.

A post hoc subgroup analysis showed that the proportion of patients with a response in the pooled groups of patients who received $15 \mathrm{mg}$ twice daily and $25 \mathrm{mg}$ twice daily (the most effective regimens) was similar among the 61 patients with the $J A K 2 \mathrm{~V} 617 \mathrm{~F}$ mutation and the 11 patients without the mutation (response rate, $51 \%$; exact $95 \%$ confidence interval [CI], 31 to 61 ; and $45 \%$; $95 \%$ CI, 23 to 83 ) as well as among 37 patients with primary myelofibrosis (response rate, $49 \%$; $95 \%$ CI, 34 to 68 ), 22 patients with post-polycythemia vera myelofibrosis (response rate, $45 ; 95 \% \mathrm{CI}, 32$ to 76 ), and 13 patients with post-essential thrombocythemia myelofibrosis (response rate, $62 \%$; 95\% CI, 14 to 68).

Twenty-four patients who received $15 \mathrm{mg}$ twice daily underwent magnetic resonance imaging (MRI) measurement of spleen and liver volume as an objective measure of response (at baseline and after one, three, and six cycles of treatment). A reduction in spleen volume detected by MRI paralleled a reduction in spleen size detected by physical examination: after 6 months of therapy, the median reduction in spleen volume was 33\% and the median reduction in spleen length was 52\% (Fig. 2 in the Supplementary Appendix). In 6 patients with hepatomegaly (an increase in liver volume that was more than two times the normal volume), the liver volume decreased by $14 \%$ after six cycles ( 6 months) of treatment.

A total of 28 patients who received $25 \mathrm{mg}$ twice daily or $15 \mathrm{mg}$ twice daily (the most active regimens for splenomegaly reduction) were transfusion-dependent at enrollment. After a median treatment duration of 12 weeks, 4 patients (14\%) had transfusion independence (clinical improvement according to the International Working Group) for a median duration of 20 months. Patients with low platelet or neutrophil counts were not eligible for participation in the study; hence, no response evaluation according to the International Working Group was possible for these variables. The mean baseline white-cell count decreased after 3 months of therapy $(\mathrm{P}=0.001)$ in patients who received $15 \mathrm{mg}$ twice daily or $25 \mathrm{mg}$ twice daily, from $29.8 \times 10^{9}$ to $16.0 \times 10^{9}$ per liter, and these counts remained stable through 1 year. Sixteen of 17 patients with elevated platelet counts at baseline (mean, $728 \times 10^{9}$ per liter) had reduced platelet counts at 3 months (mean, $336 \times 10^{9}$ per liter): of the 10 with normalized platelet counts at 3 months, 7 had counts that remained normal throughout year 1. Baseline CD34+ cell counts also decreased slightly after 3 months of therapy (from a mean of 583 to 398 cells per cubic millimeter) in 25 patients for whom serial measurements were available. 


\section{Symptomatic and Functional Improvements}

After 1 month of therapy, the majority of patients who received INCB018424 at a dose of 10 $\mathrm{mg}$ twice daily to $25 \mathrm{mg}$ twice daily had more than $50 \%$ improvement in total or individual symptom scores according to the Myelofibrosis Symptom Assessment Form (MFSAF) (on a scale of 0 to 10 , with 0 indicating no symptoms and 10 indicating maximal symptoms) (Table 3 in the Supplementary Appendix). This symptom response was durable through 6 months of therapy (Fig. 2A). Although the dose of $10 \mathrm{mg}$ twice daily was suboptimal for the reduction of splenomegaly, the improvement in symptoms with this dose was similar to the improvement associated with $15 \mathrm{mg}$ twice daily and $25 \mathrm{mg}$ twice daily (Fig. 2B). A reduction in abdominal discomfort and pain accompanied the reduction in spleen size. The mean score for pruritus (in $45 \%$ of patients who completed the MFSAF) was decreased after 2 weeks of therapy as compared with baseline ( 0.8 vs. $3.5, \mathrm{P}<0.001)$, and the improvement was maintained through 12 cycles (12 months) of therapy. Patients with myelofibrosis walk 60 to $90 \mathrm{~m}$ less than age-matched healthy controls in a 6-minute walk test. ${ }^{18}$ Twenty-seven patients in this study underwent the 6-minute walk test; an improvement in their ability to walk in this test by $34 \mathrm{~m}$ after 1 month, $57 \mathrm{~m}$ after 3 months, and $71 \mathrm{~m}$ after 6 months of therapy was observed. Study patients started to gain weight after as few as two cycles (2 months) of therapy (Fig. 2C); this weight gain was more pronounced in patients in the lowest quartile for body-mass index at baseline than in those in the highest quartile. The median weight gain after 1 year of therapy was $6.6 \mathrm{~kg}$ in patients who received $10 \mathrm{mg}$ twice daily, $9.4 \mathrm{~kg}$ in patients who received $15 \mathrm{mg}$ twice daily, and $7.1 \mathrm{~kg}$ in patients who received $25 \mathrm{mg}$ twice daily. Overall, the performance status of patients who received therapy improved over time (see the Supplementary Appendix).

\section{Effect on JAK2 V617F Allele Burden and Biologic Markers of Myelofibrosis}

At baseline, more than half the patients had a $J A K 2 \mathrm{~V} 617 \mathrm{~F}$ allele burden that was greater than $75 \%$ (Table 1). In 34 patients for whom data were available, the mean maximal suppression of $13 \%$ from baseline was seen after 12 cycles of therapy (4 patients had a 30 to $40 \%$ reduction and 1 had a decrease from 38 to 5\%). Plasma levels of various biomarkers, including several proinflammatory cytokines, were significantly higher at baseline in 23 patients who received $25 \mathrm{mg}$ twice daily than in healthy controls (Fig. 3A). Levels of leptin, a hormone derived from adipose tissue, and erythropoietin were lower than normal. After one cycle of therapy, all biomarker levels decreased, except for levels of leptin and erythropoietin, which increased. These changes were not related to the patients' JAK2 mutational status or disease subtype (Fig. 3B). Reductions in plasma levels of selected cytokines correlated with symptomatic improvements (Fig. 3C).

In addition, constitutively phosphorylated signal transducer and activator of transcription 3 (STAT3), phosphorylated signal transducer and activator of transcription 5 (STAT5), or both were observed in patients with myelofibrosis at baseline, regardless of their $J A K 2 \mathrm{~V} 617 \mathrm{~F}$ mutational status (data shown in Fig. 3A in the Supplementary Appendix). A dose- and time-dependent reduction of phosphorylated STAT3 was observed after INCB018424 treatment (Fig. 3B and 3C in the Supplementary Appendix). 


\section{Safety and Adverse Events}

Among the 153 patients enrolled in this study, the median duration of therapy was more than 1 year (>14.7 months), and 115 patients (75\%) were still receiving therapy as of this writing. Nonhematologic toxic effects related to therapy were infrequent (in $<10 \%$ of patients) and of low grade (Table 2). Hematologic toxic effects included anemia and grade 3 or 4 thrombocytopenia (Table 3). Thrombocytopenia was unrelated to JAK2 V617F mutational status, occurred more often in patients with a baseline platelet count of less than $200 \times 10^{9}$ per liter (data not shown), and was reversible within 1 to 3 weeks after dose interruption; most patients resumed treatment at a lower dose. The incidence of thrombocytopenia was significantly lower among the 35 patients who received the 15 -mg dose twice daily than among the 47 patients who received the 25-mg dose twice daily (3\% vs. 29\%), even though patients who received $15 \mathrm{mg}$ twice daily underwent subsequent dose increases to $20 \mathrm{mg}$ twice daily (approximately $25 \%$ of patients) or $25 \mathrm{mg}$ twice daily (approximately $10 \%$ of patients). In contrast, the dose was decreased or interrupted in $60 \%$ of patients who received $25 \mathrm{mg}$ twice daily because of hematologic toxicity in the first year of therapy.

Mean hemoglobin levels decreased during the first three to four cycles of therapy and then stabilized or improved with continued treatment (Fig. 4 in the Supplementary Appendix). There was a lower incidence of new-onset anemia among patients who received $15 \mathrm{mg}$ twice daily than among the patients who received $25 \mathrm{mg}$ twice daily (8\% vs. 27\%) (Table 3) and a lower proportion of patients who required transfusions ( $4.7 \%$ vs. $20 \%$ ) during therapy.

Serious adverse events occurred in 59 patients, of whom 12 had serious adverse events that were considered to be at least possibly related to treatment (Table 4 in the Supplementary Appendix). In 3 patients, there was transformation to AML. During 2 or more years of follow-up, 38 patients discontinued treatment because of withdrawal of consent, the investigator's decision to discontinue treatment, or other reasons (in 24 patients), progressive disease (in 4 patients), toxic effects (in 2 patients), intercurrent illness (in 3 patients), and death (in 5 patients) (Table 5 in the Supplementary Appendix). Seventeen of 153 patients died during the follow-up period (overall survival, 84\%) (Fig. 5A in the Supplementary Appendix), of whom 5 died during therapy and 3 died within 30 days after discontinuation of therapy (90\% survival when only deaths that occurred during the study were considered) (Fig. 5B in the Supplementary Appendix).

The pharmacokinetic and pharmacodynamic properties of therapy are described in the Supplementary Appendix.

\section{Discussion}

In this phase 1-2 study, we found that INCB018424, an oral JAK1 and JAK2 inhibitor, was associated with clinical benefits in patients with advanced (intermediate- 2 or high-risk) myelofibrosis. A starting dose of $15 \mathrm{mg}$ twice daily with individualized dose adjustment was found to be the most effective and safest dose of INCB018424. This agent was associated with rapid and sustained reduction of splenomegaly, resolution of constitutional symptoms, improvement of performance status and exercise capacity, and weight gain. These durable improvements (median follow-up, $>1$ year) were independent of $J A K 2$ mutational status or 
the cause of disease. Although a small fraction of patients with transfusion-dependent anemia became transfusion-independent, new-onset anemia developed in a similar percentage of patients, indicating that improvement in anemia is not a major benefit of therapy. Thrombocytopenia, an expected toxic effect, was defined as the dose-limiting toxic effect.

Splenomegaly and associated symptoms such as abdominal pain compromise the quality of life in patients with myelofibrosis, ${ }^{17,19}$ and alleviation of these aspects of myelofibrosis by INCB018424 is an important clinical benefit. The reduction in splenomegaly observed in most patients was not predicted, and it occurred without deterioration in hematologic variables or tumor-cell lysis. The spleen is thought to be a site of hematopoiesis in myelofibrosis; however, a reduction in spleen size did not compromise hematopoiesis. Although further investigation is needed to clarify the specific basis for the reduction in splenomegaly by therapy targeted at JAK1 and JAK2, our working hypothesis is that JAK2 inhibition is responsible for abrogation of neoplastic cell proliferation in the spleen.

Symptoms of myelofibrosis not directly related to splenomegaly, including night sweats, fevers, fatigue, weight loss, and pruritus, also improved in response to INCB018424. It has previously been reported (and confirmed here) (Fig. 3A) that patients with myelofibrosis have high levels of circulating inflammatory cytokines, such as interleukin- $6,{ }^{20}$ that probably contribute to the hypercatabolic state and constitutional symptoms such as weight loss and fatigue. ${ }^{21,22}$ Recognizing that many proinflammatory cytokines signal through a JAK1-dependent cellular pathway, we recently found that JAK1 is constitutively active in peripheral-blood cells in patients with myelofibrosis. ${ }^{12}$ A reduction in signal transduction and cytokine levels, including interleukin-6, tumor necrosis factor $a$, and macrophage inflammatory protein $1 \beta$, presumably through JAK1 and JAK2 inhibition, paralleled improvements in the patients' systemic symptoms (i.e., a decrease in the composite symptom score) after therapy (Fig. 3C).

Results demonstrating the presence of constitutively phosphorylated STAT3 that was independent of $J A K 2$ V617F mutational status (Fig. 3A in the Supplementary Appendix) provide evidence of activated JAK signaling in myelofibrosis with both wild-type and mutated JAK2 and raise the possibility of alternative pathways of JAK activation in JAK2 V617F-negative myelofibrosis. Suppression of phosphorylated STAT3 (Fig. 3B and 3C in the Supplementary Appendix) and the down-modulation of inflammatory cytokine levels and their signaling in patients with either $J A K 2 \mathrm{~V} 617 \mathrm{~F}$ or wild-type $J A K 2$ after INCB018424 treatment provide a rational biologic explanation for the finding that the response to therapy was independent of $J A K 2 \mathrm{~V} 617 \mathrm{~F}$ mutational status.

The majority of patients in this study (65\%) were in the high-risk category, according to the International Prognostic Scoring System, ${ }^{2}$ with an expected median survival of 27 months. Although the overall survival rate of $84 \%$ (90\% survival when only deaths that occurred during the study were considered) in this study with up to 2 years of follow-up is encouraging, longer follow-up and a study with a concurrent control group are needed to definitively establish any survival benefit of this therapy. On the basis of limited historical data, the expected frequency of AML transformation among patients with myelofibrosis is 
0.06 per patientyear. ${ }^{8,23}$ In the present study involving 153 patients with a median follow-up of approximately 15 months (>190 patient-years), 3 cases of transformation to AML were observed, as compared with an expected incidence of 11 cases.

In conclusion, this study shows clinical responses to a JAK1 and JAK2 inhibitor, INCB018424, in patients with advanced myelofibrosis. The clinical benefits observed with this targeted therapy suggest the potential to address important unmet medical needs in patients with myelofibrosis. On the basis of the results of this study, two controlled, phase 3 randomized trials have been initiated.

\section{Supplementary Material}

Refer to Web version on PubMed Central for supplementary material.

\section{Acknowledgments}

Funded by Incyte; ClinicalTrials.gov number, NCT00509899.

Drs. Bradley, Vaddi, Levy, Erickson-Viitanen, and Fridman report being employees of and owning stock in Incyte; Dr. Verstovsek, receiving honoraria from Incyte for participation as an advisory board member; and Dr. Kantarjian, receiving or having pending research grants from Novartis, Bristol-Myers Squibb, Wyeth, Genzyme, and Amgen, and receiving consulting fees from Novartis.

Disclosure forms provided by the authors are available with the full text of this article at NEJM.org.

We thank Dr. Tim Burn and Dr. Peggy Scherle of Incyte for pharmacodynamic and biomarker analyses, and Complete Healthcare Communications for editorial assistance with an earlier version of the manuscript.

\section{References}

1. Tefferi A. Myelofibrosis with myeloid metaplasia. N Engl J Med. 2000; 342:1255-1265. [PubMed: 10781623]

2. Cervantes F, Dupriez B, Pereira A, et al. New prognostic scoring system for primary myelofibrosis based on a study of the International Working Group for Myelofibrosis Research and Treatment. Blood. 2009; 113:2895-2901. [PubMed: 18988864]

3. Cervantes F, Passamonti F, Barosi G. Life expectancy and prognostic factors in the classic BCR/ ABL-negative myeloproliferative disorders. Leukemia. 2008; 22:905-914. [PubMed: 18385755]

4. Passamonti F, Rumi E, Pungolino E, et al. Life expectancy and prognostic factors for survival in patients with polycythemia vera and essential thrombocythemia. Am J Med. 2004; 117:755-761. [PubMed: 15541325]

5. Tefferi A. New insights into the pathogenesis and drug treatment of myelofibrosis. Curr Opin Hematol. 2006; 13:87-92. [PubMed: 16456374]

6. Kralovics R, Passamonti F, Buser AS, et al. A gain-of-function mutation of $J A K 2$ in myeloproliferative disorders. N Engl J Med. 2005; 352:1779-1790. [PubMed: 15858187]

7. Verstovsek S. Therapeutic potential of JAK2 inhibitors. Hematology Am Soc Hematol Educ Program. 2009:636-642. [PubMed: 20008249]

8. Barosi G, Bergamaschi G, Marchetti M, et al. JAK2 V617F mutational status predicts progression to large splenomegaly and leukemic transformation in primary myelofibrosis. Blood. 2007; 110:40304036. [PubMed: 17712047]

9. Tefferi A, Lasho TL, Huang J, et al. Low JAK2V617F allele burden in primary myelofibrosis, compared to either a higher allele burden or unmutated status, is associated with inferior overall and leukemia-free survival. Leukemia. 2008; 22:756-761. [PubMed: 18216871] 
10. Tefferi A, Lasho TL, Schwager SM, et al. The JAK2(V617F) tyrosine kinase mutation in myelofibrosis with myeloid metaplasia: lineage specificity and clinical correlates. Br J Haematol. 2005; 131:320-328. [PubMed: 16225651]

11. Williams DM, Kim AH, Rogers O, Spivak JL, Moliterno AR. Phenotypic variations and new mutations in JAK2 zV617F-negative polycythemia vera, erythrocytosis, and idiopathic myelofibrosis. Exp Hematol. 2007; 35:1641-1646. [PubMed: 17920755]

12. Quintás-Cardama A, Vaddi K, Liu P, et al. Preclinical characterization of the selective JAK1/2 inhibitor INCB018424: therapeutic implications for the treatment of myeloproliferative neoplasms. Blood. 2010; 115:3109-3117. [PubMed: 20130243]

13. Jaffe, ES.; Harris, NL.; Stein, H., et al. Lyon, France: IARC Press; 2001. World Health Organization classification of tumours: pathology and genetics of tumours of haematopoietic and lymphoid tissues.

14. Dupriez B, Morel P, Demory JL, et al. Prognostic factors in agnogenic myeloid metaplasia: a report on 195 cases with a new scoring system. Blood. 1996; 88:1013-1018. [PubMed: 8704209]

15. Oken MM, Creech RH, Tormey DC, et al. Toxicity and response criteria of the Eastern Cooperative Oncology Group. Am J Clin Oncol. 1982; 5:649-655. [PubMed: 7165009]

16. Tefferi A, Barosi G, Mesa RA, et al. International Working Group (IWG) consensus criteria for treatment response in myelofibrosis with myeloid metaplasia, for the IWG for Myelofibrosis Research and Treatment (IWG-MRT). Blood. 2006; 108:1497-1503. [PubMed: 16675707]

17. Mesa RA, Niblack J, Wadleigh M, et al. The burden of fatigue and quality of life in myeloproliferative disorders (MPDs): an international Internet-based survey of 1179 MPD patients. Cancer. 2007; 109:68-76. [PubMed: 17123268]

18. Mesa RA, Kantarjian H, Tefferi A, et al. Functional assessment of performance status in patients with myelofibrosis (MF): utility and feasibility of the 6-minute walk test (6MWT). J Clin Oncol. 2009; 27(Suppl):15S. abstract.

19. Mesa RA, Schwager S, Radia D, et al. The Myelofibrosis Symptom Assessment Form (MFSAF): an evidence-based brief inventory to measure quality of life and symptomatic response to treatment in myelofibrosis. Leuk Res. 2009; 33:1199-1203. [PubMed: 19250674]

20. Panteli KE, Hatzimichael EC, Bouranta PK, et al. Serum interleukin (IL)-1, IL-2, sIL-2Ra, IL-6 and thrombopoietin levels in patients with chronic myeloproliferative diseases. Br J Haematol. 2005; 130:709-715. [PubMed: 16115126]

21. Pajak B, Orzechowska S, Pijet B, et al. Crossroads of cytokine signaling - the chase to stop muscle cachexia. J Physiol Pharmacol. 2008; 59(Suppl 9):251-264.

22. Kurzrock R. The role of cytokines in cancer-related fatigue. Cancer. 2001; 92(Suppl):1684-1688. [PubMed: 11598887]

23. Guglielmelli P, Barosi G, Specchia G, et al. Identification of patients with poorer survival in primary myelofibrosis based on the burden of JAK2V617F mutated allele. Blood. 2009; 114:1477-1483. [PubMed: 19549988] 


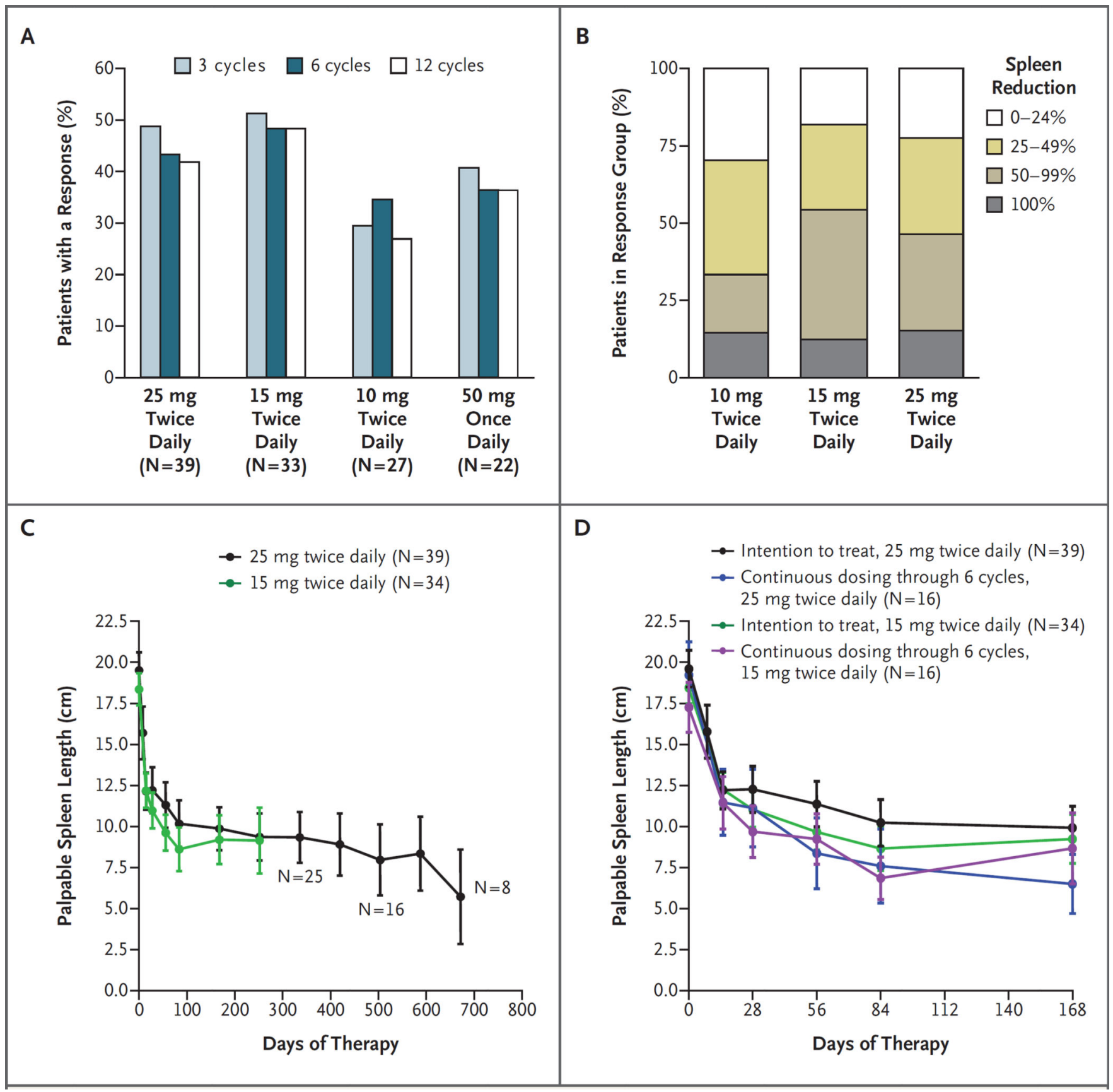

Figure 1. Clinical Responses to INCB018424 Therapy

Patients who received INCB018424 had clinical improvement (according to the criteria of the International Working Group for Myelofibrosis Research and Treatment) on the basis of a reduction of $50 \%$ or more in palpable splenomegaly, which was durable for 12 cycles (12 months) (Panel A). The proportion of patients with a 0 to $24 \%, 25$ to $49 \%, 50$ to $99 \%$, or $100 \%$ decrease in palpable spleen length relative to baseline was similar between the groups of patients in the intention-to-treat population who received $15 \mathrm{mg}$ twice daily and $25 \mathrm{mg}$ twice daily; $10 \mathrm{mg}$ twice daily provided a lesser, but durable, response (Panel B). Reduction in the spleen size was rapid and durable for approximately 2 years, as shown by the mean 
spleen size over time. The initial response to INCB018424 therapy (reduction in mean spleen length) is shown for the patients who received $15 \mathrm{mg}$ twice daily and $25 \mathrm{mg}$ twice daily (Panel C) and for the subgroup of patients who received their originally assigned dose for at least 6 months (Panel D). I bars denote standard errors. 


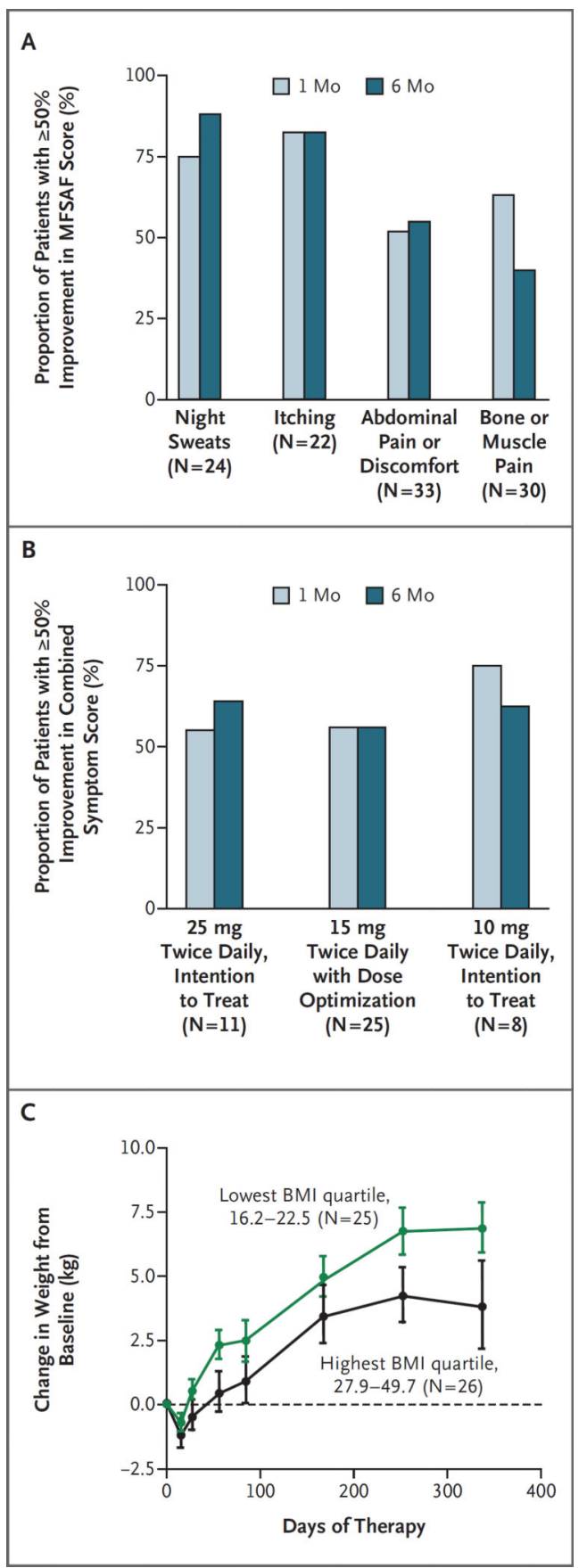

Figure 2. Effects of INCB018424 on Symptoms of Myelofibrosis and Weight

Panel A shows the proportion of patients who received INCB018424 at a dose of 10 to 25 mg twice daily who had at least a $50 \%$ decrease in the individual symptom score on the Modified Myelofibrosis Symptom Assessment Form (MFSAF) ${ }^{17}$ relative to baseline that was durable for 6 months. After 1 month, $75 \%, 82 \%, 52 \%$, and $63 \%$ of the patients had at least a 50\% improvement in scores for night sweats, itching, abdominal pain or discomfort, and bone or muscle pain, respectively. After 6 months, $88 \%, 82 \%, 55 \%$, and $40 \%$ of the patients had at least a $50 \%$ improvement in scores for night sweats, itching, abdominal pain 
or discomfort, and bone or muscle pain, respectively. A combined symptom score comprising scores for night sweats, itching, abdominal pain or discomfort, and bone or muscle pain was calculated, and the proportion of patients with a $50 \%$ or greater improvement relative to baseline was determined. As shown in Panel B, similar proportions of patients had improvement in symptoms at doses of $10 \mathrm{mg}$ twice daily (75\% at 1 month and $63 \%$ at 6 months), $15 \mathrm{mg}$ twice daily (56\% at 1 month and 56\% at 6 months), or $25 \mathrm{mg}$ twice daily (55\% at 1 month and 64\% at 6 months). As shown in Panel C, an increase in weight was observed after INCB018424 treatment in patients who received twice-daily doses of $10 \mathrm{mg}$ to $25 \mathrm{mg}$; this increase was more pronounced in patients in the lowest quartile of the body-mass index (BMI, the weight in kilograms divided by the square of the height in meters) (16.2 to 22.5) than in those in the highest quartile (27.9 to 49.7). I bars denote standard errors. 


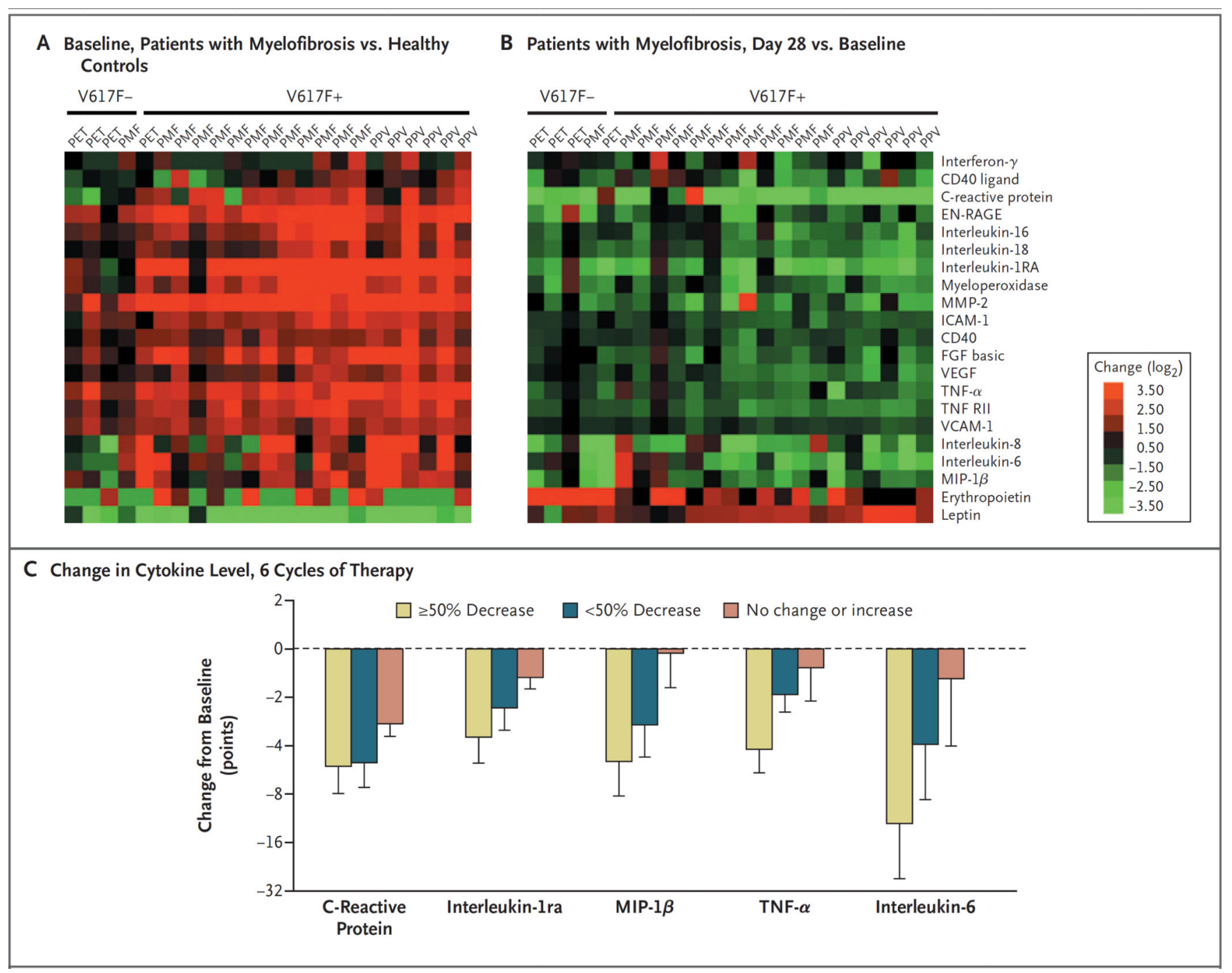

Figure 3. Effect of INCB018424 Treatment on Biomarkers

Plasma levels of various biomarkers were evaluated in samples obtained from healthy controls and patients at baseline and after one or six cycles of therapy, with the use of the HumanMAP, version 1.6 panel (Rules-Based Medicine). Plasma levels of selected markers are shown as heat maps (Panels A and B). Each row constitutes one plasma marker, with the data for individual patients organized in columns. Green and red denote markers that are present at lower and higher levels, respectively, in baseline samples from patients relative to control samples. Biomarker data obtained from patients who received INCB018424 (at a dose of $25 \mathrm{mg}$ twice daily) after one cycle of treatment were compared with baseline values for the same patients (Panel B). Green denotes markers that decreased with INCB018424 treatment, and red denotes markers that increased with therapy. Changes in selected cytokines and C-reactive protein, an acute-phase reactant and a marker for inflammation, are shown in patients with a $50 \%$ or greater decrease, those with less than a $50 \%$ decrease, and those with no change or an increase in the composite symptom score after 6 cycles of treatment as compared with baseline (Panel C). EN-RAGE denotes extracellular newly identified receptor for advanced glycosylation end products-binding protein, FGF fibroblast 
growth factor, ICAM-1 intracellular adhesion molecule 1, MIP- $1 \beta$ macrophage inflammatory protein $1 \beta$, MMP-2 matrix metalloproteinase 2, PET post-essential thrombocythemia myelofibrosis, PMF primary myelofibrosis, PPV post-polycythemia vera myelofibrosis, TNF-a tumor necrosis factor a, VCAM-1 vascular adhesion molecule 1, and VEGF vascular endothelial growth factor. 


\section{Table 1}

\section{Baseline Characteristics of the 153 Patients.}

\begin{tabular}{|c|c|}
\hline Variable & Value \\
\hline Median age (range) $-\mathrm{yr}$ & $65(40-84)$ \\
\hline \multicolumn{2}{|l|}{ Sex $-\%$ of patients } \\
\hline Male & 63 \\
\hline Female & 37 \\
\hline \multicolumn{2}{|l|}{ Risk category $-\%$ of patients ${ }^{*}$} \\
\hline Intermediate-2 & 27.5 \\
\hline High & 65.4 \\
\hline Not determined & 7.1 \\
\hline \multicolumn{2}{|l|}{ Myelofibrosis subtype $-\%$ of patients } \\
\hline Primary myelofibrosis & 53.0 \\
\hline Post-polycythemia vera myelofibrosis & 31.8 \\
\hline Post-essential thrombocythemia myelofibrosis & 15.2 \\
\hline Median time since diagnosis (range) $-\mathrm{yr}$ & $6.0(0.1-36.0)$ \\
\hline Previous myelofibrosis therapy — $\%$ of patients & 86 \\
\hline Median previous therapies (range) - no..$^{\dagger}$ & $2(0-15)$ \\
\hline Peripheral-blood blast count $\geq 1 \%-\%$ of patients & 97.5 \\
\hline$J A K 2 \mathrm{~V} 617 \mathrm{~F}-$ positive $-\%$ of patients & 82 \\
\hline Median $J A K 2 \mathrm{~V} 617 \mathrm{~F}$ allele burden (range) $-\%$ & $84(4-98)$ \\
\hline$>75 \%$ mutant allele burden $-\%$ & 61 \\
\hline Cytogenetic abnormalities $-\%$ of patients ${ }^{*}$ & 45 \\
\hline Previous transfusions - no. of patients (\%) & $52(34)$ \\
\hline Median hemoglobin (range) $-\mathrm{g} /$ liter & $104(72-169)$ \\
\hline Median platelet count (range) $-\times 10^{-9} /$ liter & $263(101-1195)$ \\
\hline Median absolute neutrophil count (range) $-\times 10^{-9} /$ liter & $12.1(1.1-135.0)$ \\
\hline Median white-cell count (range) $-\times 10^{-9} /$ liter & $17(2-202)$ \\
\hline Splenomegaly $-\%$ of patients & 92 \\
\hline Splenectomy $-\%$ of patients & 1.3 \\
\hline Median palpable spleen length (range) $-\mathrm{cm} \xi$ & $19(2-36)$ \\
\hline
\end{tabular}

The risk category ${ }^{2}$ was retrospectively determined. Risk was classified as "not determined" when all five risk assessment factors had not been evaluated or were not available.

${ }^{\dagger}$ Previous therapies used frequently included hydroxyurea, lenalidomide, prednisone, anagrelide, interferon, thalidomide, or pomalidomide. Less frequently used therapies include imatinib, CEP-701, and busulfan.

tData for 138 patients were available. Cytogenetic abnormalities frequently included deletions in 13q or 11q; other deletions or translocations occurred less frequently.

${ }^{\mathcal{B}}$ Data are for 141 patients with a palpable spleen at baseline. 


\section{Table 2}

Nonhematologic Adverse Events in 153 Patients. *

\begin{tabular}{|c|c|c|}
\hline Event & $\begin{array}{r}\text { All Grades } \\
\text { percent }\end{array}$ & $\begin{array}{l}\text { Grade } 3 \text { or } 4 \\
\text { of patients }\end{array}$ \\
\hline Diarrhea & 5.9 & 0 \\
\hline Fatigue & 4.3 & 1.3 \\
\hline Headache & 3.3 & 0 \\
\hline Peripheral edema & 2.6 & 0 \\
\hline Pain in extremities & 2.6 & 0 \\
\hline Urinary tract infection & 2.6 & 0 \\
\hline Dizziness & 2.6 & 0 \\
\hline Dyspnea & 2.6 & 0 \\
\hline Asthenia & 2.0 & 2.0 \\
\hline Fever & 2.0 & 0.7 \\
\hline Cardiac murmur & 2.0 & 0 \\
\hline Musculoskeletal pain & 2.0 & 0 \\
\hline Peripheral neuropathy & 2.0 & 0 \\
\hline Edema & 2.0 & 0 \\
\hline Anxiety & 2.0 & 1.3 \\
\hline Insomnia & 2.0 & 1.3 \\
\hline Epistaxis & 2.0 & 0 \\
\hline Flatulence & 2.0 & 0 \\
\hline Nausea & 2.0 & 0 \\
\hline
\end{tabular}

Adverse events that were thought to be at least possibly related to the study medication and that occurred in $2 \%$ or more of the study population are included. 


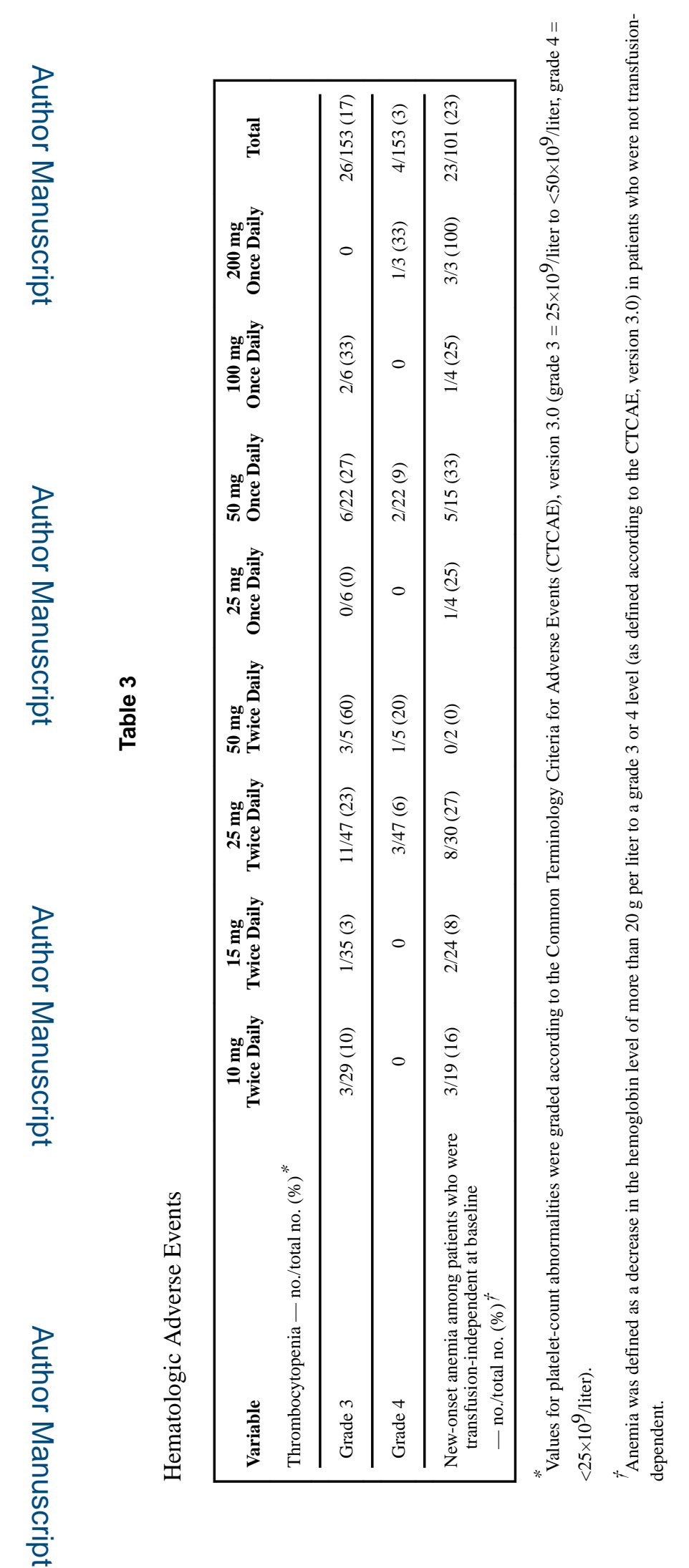

NEngl J Med. Author manuscript; available in PMC 2016 December 27. 